\title{
Hidden landscape of an Anderson insulator
}

\author{
Computing spectra without solving eigenvalue problems \\ Authors: Douglas Arnold, Guy David, Marcel Filoche, David Jerison, Svitlana \\ Mayboroda \\ SIAM J. Sci. Comput. 41, B69 (2019) [arXiv:1711.04888]
}

\section{Recommended with a Commentary by Carlo Beenakker, Instituut-Lorentz, Leiden University}

Anderson localization in a random potential is very much a 20th century topic, and one would not expect the 21st century to bring conceptually new insights. But that is what the paper by Arnold et al. offers, developing the concept of a "localization landscape" pioneered by Filoche and Mayboroda (PNAS, 2012).

While the concept is far-reaching, it can be introduced in a few lines of basic math. Any eigenfunction $\psi$ at eigenvalue $E>0$ of an invertible Hermitian operator $H$ satisfies the inequality

$$
|\psi(\boldsymbol{r})| \leq E|\psi|_{\max } u(\boldsymbol{r}), \text { with } u(\boldsymbol{r})=\int\left|H^{-1}\left(\boldsymbol{r}, \boldsymbol{r}^{\prime}\right)\right| d \boldsymbol{r}^{\prime},
$$

which follows from $\psi=E H^{-1} \psi$ and $|\psi(\boldsymbol{r})| \leq|\psi|_{\max }$. If $H=-\frac{\hbar^{2}}{2 m} \nabla^{2}+V$ is the single-electron Hamiltonian in a potential $V(\boldsymbol{r})>0$, the Green's function $H^{-1}\left(\boldsymbol{r}, \boldsymbol{r}^{\prime}\right)>0$ is positive ${ }^{1}$ and the absolute value signs in the definition of $u$ can be omitted. This function can then be obtained from a single differential equation,

$$
\left[-\frac{\hbar^{2}}{2 m} \nabla^{2}+V(\boldsymbol{r})\right] u(\boldsymbol{r})=1,
$$

without having to calculate the full Green's function. The spatial dependence of $u$ is referred to as the localization landscape. ${ }^{2}$

The localization landscape $u(\boldsymbol{r})$ is fully determined by the potential landscape $V(\boldsymbol{r})$, but in a highly convoluted way - which is why it is referred to as a "hidden landscape". Figure 1 illustrates this for a one-dimensional random potential. The spatial location of the lowest eigenstates lines up with the highest peaks of the localization landscape (right panel), but the correlation with the potential landscape (left panel) is much less evident. This is the essence of quantum localization, which is due to the destructive interference of multiply scattered waves rather than to the confinement by potential barriers.

\footnotetext{
${ }^{1}$ This follows from the maximum principle for elliptic operators. Note that we need pointwise positivity of $H^{-1}\left(\boldsymbol{r}, \boldsymbol{r}^{\prime}\right)$, positivity of the eigenvalues of $H^{-1}$ is not sufficient.

${ }^{2}$ This terminology is due to Filoche and Mayboroda (PNAS, 2012). The function $u$ for $V=0$ is called the torsion function in elasticity theory (arXiv:1510.06353), where it plays a role in shape optimization problems. In that context localization by tunnel barriers is called "weak localization" — which explains the puzzling title of their 2012 paper.
} 

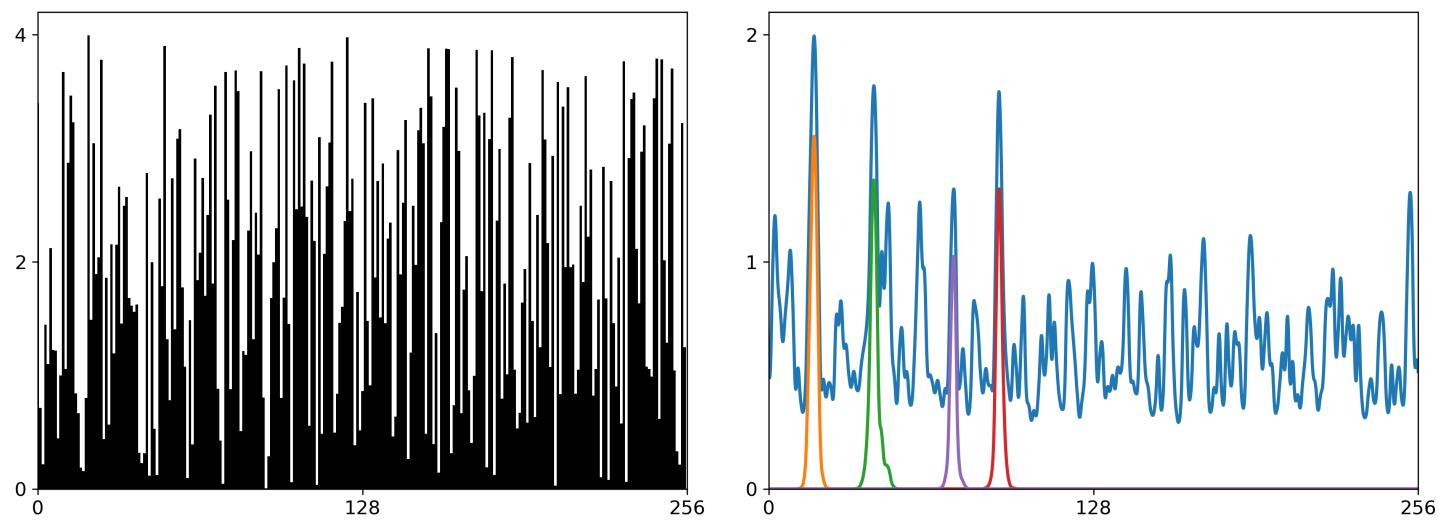

Fig. 1. The potential function $V(x)$ in the left panel gives rise to the landscape function $u(x)$ plotted in blue in the right panel. The first four eigenfunctions are also plotted, scaled so that their maximum value is the reciprocal of their eigenvalue - to demonstrate the inequality $|\psi| \leq u$ when $E|\psi|_{\max }=1$. The pseudopotential $W$ is the reciprocal of $u$. [Figure from Arnold et al. (2019).]

The reciprocal $W=1 / u$ has the dimension of a potential energy, it is a smoothed version of the original potential $V$ - one might call $W$ a pseudopotential. ${ }^{3}$ The smoothing produces local minima separated by extended barriers. In an earlier paper (PRL, 2016) Arnold et al. showed that a wave function at energy $E$ decays exponentially into the barrier regions where $W(\boldsymbol{r})>E$. This is a nontrivial result, because the inequality

$$
|\psi(\boldsymbol{r})| \leq \frac{E}{W(\boldsymbol{r})}|\psi|_{\max }
$$

would have also allowed a slow decay. In a sense the transformation from $V$ to $W$ transforms quantum localization into a classical localization problem with the same electron density profile.

The 2019 paper extends the correspondence from eigenfunctions to eigenvalues: Each local minimum $W_{\min }$ of the pseudopotential corresponds to a localized eigenstate at energy $E \approx c W_{\min }$ for some constant $c$ of order unity. This is again nontrivial, because the inequality (3) would only imply $E \geq W_{\min }$ - rather than a correspondence for a range of eigenvalues with a single coefficient $c$. The coefficient does depend on the spatial dimensionality $d$. There is no precise calculation of $c$, but a qualitative estimate of the lowest eigenvalue in a potential well of $W(\boldsymbol{r})$ gives $c \approx 1+d / 4$. Figure 2 shows how accurately the correspondence

$$
E=(1+d / 4) W_{\min }
$$

works out, for $d=1$ and $d=2$.

To appreciate how astonishing this is, imagine someone telling you of a new method to find the eigenvalues of a large random matrix: "I invert the matrix, sum the rows to obtain a column vector, and then search for local minima in its reciprocal elements." This sounds like a crazy method, but Figure 2 shows it works for localized eigenstates.

\footnotetext{
${ }^{3}$ Chandra Varma reminded me of the pseudopotential method in band structure calculations, where it refers to a smoothing of the ionic potential.
} 

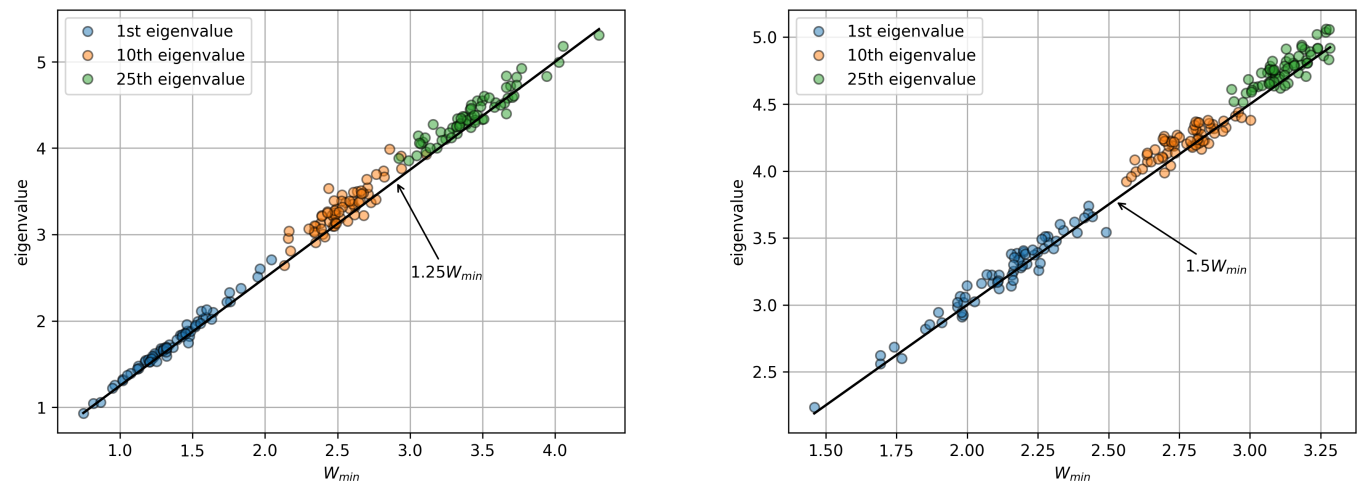

Fig. 2. The 1st, 10 th, and 25 th eigenvalues versus the corresponding minima $W_{\min }$ of the pseudopotential, for 64 realizations of a random potential. The left panel is for dimensionality $d=1$, the right panel for $d=2$. The solid line is the prediction from Eq. (4). [Figure from Arnold et al. (2019).]

An analytical, rather than numerical, justification is based on the transformation of the original Hamiltonian $H=-\frac{\hbar^{2}}{2 m} \nabla^{2}+V$ into a new operator $\tilde{H}=-\frac{\hbar^{2}}{2 m} L+W$ having the same eigenvalues, with eigenfunctions related by $\tilde{\psi}=W \psi$. The differential operator $L=W^{2} \nabla W^{-2} \nabla$ is more complicated than the original Laplacian, but Arnold et al. are able to employ existing methods for elliptic operators (notably the notion of Agmon distance ${ }^{4}$ ) to show that eigenstates of $\tilde{H}$ decay exponentially into regions where $W>E$. The eigenvalue relation (4) is on less rigorous footing.

A practical merit of the new approach to wave localization is the reduction in computational complexity. The localization landscape $u(\boldsymbol{r})$ is a smooth solution of the elliptic partial differential equation (2), which can be obtained in a time that scales linearly with the system size $L$. Efficient eigenvalue solvers scale at least $\propto L^{2}$. An application to the study of localized vibrations in enzymes which appeared recently (arXiv:1902.09939) reports a 50-fold reduction in computational time compared to conventional methods. Because the mapping from $V$ to $u$ is easy to calculate it might be feasible to engineer localized waves at specific locations by inversion of the mapping, starting from a pseudopotential $W=1 / u$ with deep valleys at the desired locations.

The conceptual merit of the approach is still very much open-ended. Might it be possible to describe the Anderson metal-insulator transition by a percolation transition in the localization landscape? Is the fractal character of wave functions at the Anderson transition reflected in the spatial statistics of the pseudopotential?

All of this is single-electron physics, but Anderson localization plays a role as well in interacting systems. In a very recent development, Balasubramanian, Liao, and Galitski (arXiv:1908.05283) have applied the notion of a localization landscape to the many-particle Fock space. The many-body localization landscape is defined for a wide class of interacting Hamiltonians on a graph whose nodes represent the Fock states, while the edges give tran-

\footnotetext{
${ }^{4}$ The Agmon distance $d_{A}$ is computed like an optical path length for a refractive index $n(\boldsymbol{r})=$ $\sqrt{\max (0, W(\boldsymbol{r}) / E-1)}$. The decay of an eigenstate at energy $E$ is bounded by $e^{-d_{A} \sqrt{2 m E / \hbar^{2}}}$.
} 
sitions due to the creation or annihilation of particle-hole excitations. Also in that context, the original problem of localization by quantum interference is mapped onto localization by tunnel barriers. We can expect to see more applications of this revolutionary idea.

I have benefited from interactions with Victor Galitski on MathOverflow. 